\title{
Parásitos intestinales en habitantes de una comunidad rural del Estado Bolívar, Venezuela.
}

\section{Comunicación Breve}

Rodolfo Devera, Verónica Angulo, Ernesto Amaro, Miguel Finali, Gabriela Franceschi, Ytalia Blanco, Rosa María Tedesco, Ixora Requena, Virma Velásquez.

Grupo de Parasitosis Intestinales, Dpto. de Parasitología y Microbiología de la Escuela de Ciencias de la Salud, Universidad de Oriente, Ciudad Bolívar, Estado Bolívar, Venezuela.

\section{RESUMEN.}

Introducción. Las parasitosis intestinales constituyen un importante problema de salud pública por sus altas tasas de prevalencia y amplia distribución mundial, sobre todo en las regiones tropicales y subtropicales, siendo la población infantil la mayormente afectada.

Objetivo. Determinar la prevalencia de parásitos intestinales en habitantes de Aripao, comunidad rural del estado Bolívar, Venezuela.

Materiales y Métodos. En abril de 2005 se realizó un estudio transversal con 155 habitantes de ambos sexos. De cada uno se obtuvo una muestra fecal la cual fue analizada mediante la técnica de examen directo, métodos de concentración de Kato y formol-éter, cultivo en placa de agar y coloración de Kinyoun.

Resultados. La prevalencia de parasitosis intestinal fue de $83.9 \%(130 / 155)$. Las prevalencias por grupos de edad fueron similares $\left(\chi^{2}=3.037\right.$; g.l.
$=5)$. Ambos sexos fueron afectados por igual ( $\mathrm{p}>$ 0.05). Se diagnosticaron 15 especies de parásitos y/o comensales. Los protozoarios fueron más prevalentes que los helmintos. Blastocystis hominis fue el parásito intestinal más prevalente con 60\%. Se diagnosticaron 9 casos de Cryptosporidium parvum y 2 de Cyclospora cayetanensis. Entre los helmintos Ascaris lumbricoides (20\%), Trichuris trichiura (9\%) y Strongyloides stercoralis (4.5\%) resultaron los más comunes. De los parasitados $75.4 \%$ resultó poliparasitado (98/130). En este último grupo los parásitos más frecuentemente asociados fueron Blastocystis hominis (75.5\%) y Entamoeba coli (48.9\%).

Conclusión. Se determinó una elevada prevalencia (83.9\%) de parásitos intestinales en habitantes de la comunidad rural de Aripao, estado Bolívar, Venezuela, sin predilección por el sexo o la edad y con predominio de los protozoarios, en particular de B. hominis. (Rev Biomed 2006; 17:259-268)

Solicitud de sobretiros: Dr. Rodolfo Devera. Departamento de Parasitología y Microbiología, Escuela de Ciencias de la Salud, Universidad de Oriente, Núcleo Bolívar. Av. José Méndez. Ciudad Bolívar, Estado Bolívar, Código postal: 8001-A. Venezuela.

Correo electrónico: rodolfodevera@hotmail.com

Recibido el 31/Agosto/2006. Aceptado para publicación el 28/Noviembre/2006.

Este artículo está disponible en http://www.uady.mx/sitios/biomedic/revbiomed/pdf/rb061744.pdf

Vol. 17/No. 4/Octubre-Diciembre, 2006 
R Devera, V Angulo, E Amaro, M Finali, G Franceschi, Y Blanco, R Maria Tedesco y col.

Palabras clave: parásitos intestinales, epidemiología, Blastocystis hominis.

\section{SUMMARY.}

Intestinal parasites in inhabitants from a rural community in Bolivar State, Venezuela.

Introduction. Intestinal parasitosis constitutes an important public health problem, due to its high prevalence rate and world-wide distribution, mainly in the tropical and subtropical regions. Theinfantile population being the most affected.

Objective. To determine the prevalence of intestinal parasites in inhabitants from Aripao, a rural community of Bolivar State, Venezuela.

Material and Methods.Across-sectional study was carried out in April 2005 on 155 inhabitants of either sex. A stool sample was obtained from each person and analyzed by the direct examination technique, the Kato and formol-ether concentration methods, agar-plate culture, and Kinyoun staining.

Results. The prevalence of intestinal parasites was of $83.9 \%(130 / 155)$. Prevalence values by age groups were similar $\left(\chi^{2}=3.037\right.$; d.f. $\left.=5\right)$. Both sexes were affected equally ( $p>0.05) .15$ species of parasites and/or commensals were diagnosed. Protozoans were more prevalent than helminths. Blastocystis hominis was the most common parasite with $60 \%$ prevalence. Nine cases of Cryptosporidium parvum and 2 cases of Cyclospora cayetanensis were diagnosed. Among the helminths, Ascaris lumbricoides (20\%) Trichuris trichiura (9\%), and Strongyloides stercoralis (4.5\%) were the most common. $75.4 \%$ of the infected subjects had more than one parasite (98/130). In this last group the parasites frequently associated were Blastocystis hominis (75.5\%) and Entamoeba coli (48.9\%).

Conclusion. A high prevalence (83.9\%) of intestinal parasites was determined in the inhabitants of the rural community of Aripao, Bolivar State, Venezuela, with no preference for age or sex and with a high proportion of protozoans, especially $B$. hominis.
(Rev Biomed 2006; 17:259-268)

Key words: intestinal parasites, epidemiology, Blastocystis hominis.

\section{INTRODUCCIÓN.}

Las infecciones por parásitos intestinales constituyen un importante problema de salud pública por sus altas tasas de prevalencia y amplia distribución mundial, sobre todo en las regiones tropicales y subtropicales, siendo la población infantil la mayormente afectada (1-3).

Entre los factores que favorecen la adquisición y desarrollo de este tipo de infecciones se encuentra el incremento de la densidad poblacional en las zonas rurales, deficientes condiciones sanitarias, bajo nivel socioeconómico, mala disponibilidad de agua, deficiente eliminación de excretas y el clima tropical. Igualmente, vivir en zonas endémicas o viajar a éstas, mala higiene personal, comer alimentos contaminados, vivir en hacinamiento y toda una serie de factores tanto sociales como económicos que en conjunto constituyen la causa social de las infecciones por parásitos $(4,5)$.

Las parasitosis intestinales representan un marcador de atraso socio-cultural; además, constituyen un índice de contaminación fecal. Sin embargo, lo más preocupante es que sus prevalencias han variado poco en los últimos 60 años en América Latina, permaneciendo elevadas (4).

Antes de implementar las posibles medidas de control es necesario conocer los determinantes locales de estas enfermedades. De allí la importancia de realizar estudios epidemiológicos sobre el problema (6).

Por razones ecológicas, epidemiológicas, sociales, sanitarias y hasta económicas las poblaciones rurales son más susceptibles de tener parasitosis intestinales (4).

Diversos estudios realizados en comunidades rurales en América Latina (4, 7, 8), Venezuela (911) y el estado Bolívar (12-14) han mostrado

\section{Revista Biomédica}


prevalencias elevadas.

Considerando lo anterior, se decide realizar una investigación para determinar la prevalencia de parásitos intestinales en habitantes de Aripao, comunidad rural del estado Bolívar, Venezuela, y contribuir de esta forma al conocimiento de los factores epidemiológicos de las parasitosis intestinales en nuestra región.

\section{MATERIAL Y MÉTODOS.}

\section{1. Área de Estudio.}

El municipio Sucre se ubica al noroeste de la capital del estado Bolívar, limita al norte con el Río Orinoco, al sur con Brasil, al oeste con el municipio Cedeño, y al este con los municipios Héres y Raúl Leoni. La Parroquia Aripao tiene una población de 769 habitantes según censo suministrado por la alcaldía del municipio Sucre. Esta población se distribuye en una comunidad central de 315 habitantes y otras pequeñas comunidades ubicadas periféricamente que constituyen el total de la parroquia. Aripao es una comunidad rural ubicada a 278 km de Ciudad Bolívar, capital del estado Bolívar. Se ubica en la margen derecha del río Caura, a 5 km de Maripa, capital del municipio Sucre. Las actividades económicas fundamentales son la pesca y la agricultura. Muchos de sus habitantes trabajan en la vecina población de Maripa.

\section{Universo y Muestra.}

El universo lo constituyeron los 315 habitantes de la comunidad y la muestra estuvo formada por todas las personas que aceptaron participar y que eran residentes permanentes en la comunidad.

Para motivar la participación de las personas se realizaron visitas a la comunidad, se conversó con los lideres de ella (enfermeras, maestras, etc.). También se informó y solicitó colaboración a las autoridades políticas y sanitarias del municipio.

\section{Recolección de Datos.}

La investigación fue de tipo transversal y consistió en la recolección de muestras fecales en abril de 2005.

\subsection{Datos de identificación y epidemiológicos.}

Después de obtener el consentimiento por escrito de cada habitante participante se investigaron datos de identificación y epidemiológicos de interés. Los mismos fueron anotados en un instrumento de recolección de datos.

\subsection{Recolección de heces.}

Se les explicó a los habitantes el procedimiento para la recolección de las heces y luego se les entregó el envase recolector apropiado.

\subsection{Análisis de las muestras fecales.}

Las muestras fecales frescas obtenidas por evacuación espontánea fueron sometidas a las técnicas del examen directo, método de Kato, cultivo en placa de agar y coloración de Kinyoun (Botero y Restrepo, 1998). Posteriormente una porción de la muestra fue preservada en formol al $10 \%$ y analizada mediante la técnica de formoléter (15) en el Laboratorio del Departamento de Parasitología y Microbiología.

\section{Análisis de datos}

Con la información obtenida se construyó una base de datos con el auxilio del programa SPSS versión 8.0 para Windows. Para el análisis de los resultados se utilizaron frecuencias relativas (\%). También se usó la prueba ji al cuadrado $\left(\chi^{2}\right)$ con un intervalo de confianza de 95\% para demostrar la independencia entre las variables: parasitosis, sexo y edad (16).

\section{RESULTADOS.}

Fueron estudiadas 155 muestras fecales provenientes de igual número de individuos de ambos sexo y de todas las edades. La media de edad fue de 20.3 años con una desviación estándar de 18.4. Un total de 92 (59.4\%) eran del sexo femenino y 63 (40.6\%) del sexo masculino.

La prevalencia de parásitos intestinales fue de 83.9\% (130/155). Todos los grupos etarios fueron afectados por igual $\left(\chi^{2}=3.037\right.$ g.l. $\left.=5 \quad \mathrm{p}>0.05\right)$ (cuadro 1). Con relación al sexo, 83.7\% del sexo 
R Devera, V Angulo, E Amaro, M Finali, G Franceschi, Y Blanco, R Maria Tedesco y col.

Cuadro 1

Habitantes con y sin parásitos según edad, comunidad rural de Aripao, Estado Bolívar, Venezuela, 2005.

\begin{tabular}{|c|c|c|c|c|c|c|}
\hline \multirow{3}{*}{$\begin{array}{l}\text { Edad } \\
\text { (Años) }\end{array}$} & \multicolumn{4}{|c|}{ Habitantes } & \multirow{2}{*}{\multicolumn{2}{|c|}{ Total }} \\
\hline & \multicolumn{2}{|c|}{ Parasitados } & \multicolumn{2}{|c|}{ No Parasitados } & & \\
\hline & $\mathbf{N}^{\mathbf{0}}$ & $\%$ & $\mathbf{N}^{\mathbf{o}}$ & $\%$ & $\mathbf{N}^{\mathbf{0}}$ & $\%$ \\
\hline $0-9$ & 45 & 86.5 & 7 & 13.5 & 52 & 33.5 \\
\hline $10-19$ & 42 & 82.3 & 9 & 17.6 & 51 & 32.9 \\
\hline $20-29$ & 11 & 91.6 & 1 & 8.3 & 12 & 7.7 \\
\hline $30-39$ & 13 & 86.6 & 2 & 13.3 & 15 & 9.8 \\
\hline $40-49$ & 6 & 66.6 & 3 & 33.3 & 9 & 5.8 \\
\hline$\geq 50$ & 13 & 81.3 & 3 & 18.8 & 16 & 10.3 \\
\hline TOTAL & 130 & 83.9 & 25 & 16.1 & 155 & 100.0 \\
\hline
\end{tabular}

femenino resultó parasitado, mientras que de la población masculina $84.1 \%$ estaba parasitado (p $>0.05$ ).

Un total de 15 especies de enteroparásitos fueron diagnosticadas, siendo los protozoarios los más frecuentes (96.2\%). En este grupo los más prevalentes fueron Blastocystis hominis (60.0\%) y Entamoeba coli (34.2\%). Cabe destacar la presencia de siete casos de Cryptosporidium parvum (7.1\%) y dos de Cyclosporra cayetanensis (2\%). Entre los helmintos Ascaris lumbricoides (20\%), Trichuris trichiura (9\%) y Strongyloides stercoralis (4.5\%) resultaron los más prevalentes (cuadro 2).

De los infectados, 24.6\% (32/130) resultó monoparasitado, mientras que 75.4\% (98/130) estaba poliparasitado. En este último grupo los parásitos más frecuentemente asociados fueron los protozoarios, siendo el más común $B$. hominis con $75.5 \%$, seguido de E. coli con 48.9\%. Entre los helmintos los más comúnmente asociados fueron A. lumbricoides con $19.3 \%$ seguido de T. trichiura con $13.3 \%$ (cuadro 3).

La prevalencia global de parásitos varió según la técnica empleada, siendo el examen directo la que proporcionó mejores resultados (72.3\% de prevalencia). En el caso de los helmintos tanto totales como los geohelmintos la técnica de Kato ofreció mejores resultados ya que la prevalencia fue de 28.45 y $27.7 \%$, respectivamente; mientras que con las otras técnicas (examen directo y formol-éter) la prevalencia fue de 14.2\%. Cuando se consideran los geohelmintos más comunes ese hallazgo se repite, pues en el Kato la prevalencia de A. lumbricoides y T. trichiura fue de $18.1 \%$ y $6.5 \%$, respectivamente; mientras que en el directo fue de $10.3 \%$ y 3.3 , respectivamente; en el formoléter se obtuvo $11.6 \%$ para A. lumbricoides y $1.9 \%$ para T. trichiura. Finalmente, para la identificación de los protozoarios el examen directo (50.3\%) y el formol-éter (51\%) ofrecieron prevalencias similares, sin embargo, cuando se evalúa la prevalencia de $B$. hominis se verifica que el examen directo (50.3\%) supera a la técnica de formol-éter (28.4\%) (cuadro 4). Se excluyeron de este análisis los resultados obtenidos con la placa de agar por ser una técnica específica para el diagnóstico de S. stercoralis.

\section{DISCUSIÓN.}

Se determinó una elevada prevalencia de parasitosis intestinales (83.9\%) en la comunidad rural de Aripao. Esta cifra coincide con aquellas obtenidas por varios investigadores en diferentes zonas del país (9-11, 17-20) y del estado Bolívar (12-14). Estas elevadas tasas de infección en general son un reflejo de la situación en la que

\section{Revista Biomédica}


Parásitos intestinales en una comunidad rural

\section{Cuadro 2}

Prevalencia de parásitos intestinales en habitantes de la comunidad rural de Aripao, estado Bolívar, Venezuela, 2005.

\begin{tabular}{lcc}
\hline \multicolumn{1}{c}{ ESPECIES } & $\mathbf{N}^{\mathbf{0}}$ & $\mathbf{\%}$ \\
\hline \multicolumn{1}{c}{ PROTOZOARIOS } & & \\
Blastocystis hominis & 93 & 60.0 \\
Entamoeba coli & 53 & 34.2 \\
Endolimax nana & 35 & 22.6 \\
Giardia lamblia & 34 & 21.9 \\
Entamoeba histolytica/E. dispar & 18 & 11.6 \\
Iodamoeba butschlii & 15 & 9.7 \\
Cryptosporidium parvum $\quad$ Chilomastix mesnilii & 9 & 5.8 \\
\multicolumn{1}{c}{ Pentatrichomonas hominis } & 6 & 3.9 \\
$\quad 3$ & 1.9 \\
Hyclospora cayetanensis* & 2 & 1.3 \\
$\quad$ Ascaris lumbricoides & 2 & \\
Trichuris trichiura & 31 & 20.0 \\
Strongyloides stercoralis $\dagger$ & 14 & 9.0 \\
Hymenolepis nana & 7 & 4.5 \\
Ancilostomideos & 4 & 2.6 \\
\hline
\end{tabular}

* Empleando coloración de Kinyoun

† Empleando cultivo en placa de agar

viven los habitantes de este tipo de comunidades, entre ellas saneamiento ambiental deficiente y condiciones socioeconómicas precarias (20).

Ambos sexos fueron afectados por igual, coincidiendo este hallazgo con la mayoría de los estudios realizados sobre parasitosis en comunidades rurales y suburbanas $(10,14,19$, 20). Y no hubo diferencias con relación a las parasitosis y los grupos de edad. Posiblemente debido a que todos están expuestos a los mismos factores que determinan las parasitosis o tienen los mismos hábitos higiénicos y, por consiguiente, la población es afectada por igual. Ese hallazgo coincide con el de otros autores $(10,11)$.

Como en otros estudios, el poliparasitismo fue un hallazgo común, lo que demuestra un elevado nivel de transmisión debido a que existen las condiciones para ello $(10,19,20)$. Los habitantes están de forma persistente expuestos a los ambientes contaminados con parásitos, lo que permite la posibilidad de adquirir un amplio espectro de especies parasitarias ya que muchos de estos parásitos comparten la misma epidemiología, por lo que en su transmisión juegan un papel importante las condiciones ecológicas y de medio ambiente, además de las deficiencias en los hábitos de higiene $(20,21)$.

Hubo un predominio de protozoarios sobre los helmintos, hecho que se ha venido observando en los últimos años tanto en Venezuela (10, 19, 20) como en el estado Bolívar (13, 22, 23). Posiblemente el uso de drogas antiparasitarias de amplio espectro pero con acción fundamentalmente antihelmintica sea una de las razones que explican ese predominio de protozoarios. La otra puede ser la deficiencia en el suministro y almacenamiento de agua potable.

El parásito más prevalente fue $B$. hominis (60.0\%), lo cual coincide con la mayoría de los estudios realizados en la última década en diversos grupos poblacionales de varias partes del mundo que revelan que se trata de un patógeno emergente de elevada prevalencia aunque de patogenicidad discutida (7, 8, 24). En Venezuela B. hominis también es actualmente el enteroparásito más común $(9-11,20)$. En el Estado Bolívar la Vol. 17/No. 4/Octubre-Diciembre, 2006 
R Devera, V Angulo, E Amaro, M Finali, G Franceschi, Y Blanco, R Maria Tedesco y col.

Cuadro 3

Parásitos asociados en 98 habitantes poliparasitados, Aripao, estado Bolívar, Venezuela, 2005.

\begin{tabular}{|c|c|c|}
\hline ESPECIES & $\mathbf{N}^{\mathbf{0}}$ & $\%$ \\
\hline \multicolumn{3}{|l|}{ PROTOZOARIOS } \\
\hline Blastocystis hominis & 74 & 75.5 \\
\hline Entamoeba coli & 48 & 48.9 \\
\hline Endolimax nana & 31 & 31.6 \\
\hline Giardia lamblia & 31 & 31.6 \\
\hline Entamoeba histolytica/E. dispar & 17 & 17.3 \\
\hline Iodamoeba butschlii & 12 & 12.2 \\
\hline Cryptosporidium parvum* & 7 & 7.1 \\
\hline Chilomastix mesnilii & 6 & 6.1 \\
\hline Pentatrichomonas hominis & 2 & 2.0 \\
\hline Cyclospora cayetanensis* & 2 & 2.0 \\
\hline \multicolumn{3}{|l|}{ HELMINTOS } \\
\hline Ascaris lumbricoides & 19 & 19.3 \\
\hline Trichuris trichiura & 13 & 13.3 \\
\hline Hymenolepis nana & 4 & 4.1 \\
\hline Strongyloides stercoralis $\dagger$ & 3 & 3.1 \\
\hline Ancilostomideos & 6 & 6.1 \\
\hline
\end{tabular}

prevalencia de $B$. hominis también es alta, tanto en el medio urbano $(23,24)$ como en el rural $(13$, 14, 25).

Después de $B$. hominis, los protozoarios comensales Entamoeba coli y Endolimax nana destacaron por su prevalencia relativamente alta.
Si bien su identificación carece de importancia clínica, tiene gran significado epidemiológico pues su presencia indica contaminación fecal del agua y/o alimentos en la comunidad evaluada $(17,20)$.

Giardia lamblia ocupó el primer lugar dentro de los protozoarios patógenos con una prevalencia

\section{Cuadro 4}

Prevalencia de los principales helmintos y protozoarios utilizando diferentes técnicas diagnósticas en habitantes de Aripao, estado Bolívar, Venezuela, 2005.

\begin{tabular}{|l|c|c|c|c|c|c|}
\hline & \multicolumn{6}{c|}{ Técnica diagnóstica } \\
\hline \multicolumn{1}{|c|}{ PARÁSITOS } & \multicolumn{2}{|c|}{ ED } & \multicolumn{2}{c|}{ Kato } & \multicolumn{2}{c|}{ Formol éter } \\
\hline & No. & $\mathbf{\%}$ & No. & $\mathbf{\%}$ & No. & $\mathbf{\%}$ \\
\hline Parásitos totales & 112 & 72.3 & 35 & 22.6 & 90 & 50.1 \\
\hline Helmintos Totales & 22 & 14.2 & 44 & 28.4 & 22 & 14.2 \\
\hline Geohelmintos totales & 22 & 14.2 & 43 & 27.7 & 22 & 14.2 \\
\hline Ascaris lumbricoides & 16 & 10.3 & 28 & 18.1 & 18 & 11.6 \\
\hline Trichuris trichiura & 5 & 3.2 & 10 & 6.5 & 3 & 1.9 \\
\hline Protozoarios totales & 86 & 55.5 & NA & NA & 79 & 51.0 \\
\hline Blastocystis hominis & 78 & 50.3 & NA & NA & 44 & 28.4 \\
\hline
\end{tabular}

ED = Examen directo

NA $=$ No aplicable

\section{Revista Biomédica}




\section{Parásitos intestinales en una comunidad rural}

de 21.9\%. Años atrás este era el principal protozoario diagnosticado en el estado Bolívar, siendo actualmente desplazado por $B$. hominis (22, 24).

La prevalencia de coccidios intestinales fue relativamente baja (7.1\% para $C$. parvum y $2 \%$ para C. cayetanensis). Como en otros estudios C. parvum fue el coccidio intestinal más común (23, 26, 27). En comunidades urbanas de Ciudad Bolívar se ha informado de prevalencias similares (23) y también con predominio de $C$. parvum. Aunque en otras regiones del país esas cifras han sido mayores (28-30). Recientemente Devera et al. (31) determinaron una elevada prevalencia de $C$. cayetanensis (13\%) entre indígenas de una comunidad al sur del estado Bolívar. Sin embargo, ese ha sido el único estudio donde Cyclospora ha superado en prevalencia a Cryptosporidium. Las prevalencias aquí encontradas son inferiores a las señaladas en pacientes con diarrea, en menores de 5 años y en inmunosuprimidos, tanto en Venezuela (30, 32-34) como en el estado Bolívar (35).

Aunque se esperaba encontrar una elevada prevalencia de geohelmintos debido a las características sanitarias y eco-epidemiológicas de la comunidad, apenas se obtuvo un $20 \%$ para A. lumbricoides y $9 \%$ para $T$. trichiura. Las asociaciones entre ambas fueron muy infrecuentes aun cuando existe similitud marcada en la epidemiología de ambas parasitosis $(17,20$, 21, 36). En el estado Bolívar, principalmente en comunidades rurales, $T$. trichiura ha sido señalado como el helminto más común $(12,22)$, siendo su prevalencia similar a la de A. lumbricoides, aunque en los últimos años la cifra de esta última ha disminuido $(21,22,36)$.

Con respecto a otros helmintos, destacaron los siete casos de $S$. stercoralis. Esta prevalencia coincide con los resultados encontrados por otros investigadores en el Estado Bolívar (22, 36), así como en otras partes de Venezuela (37).

En cuanto a las asociaciones parasitarias, en la mayor parte de ellas se encontraba un protozoario acompañando de otro protozoario. Blastocystis hominis y E. coli resultaron los parásitos más frecuentemente asociados, coincidiendo con varios estudios realizados en el estado Bolívar $(14,20,23,24)$. Las asociaciones parasitarias entre geohelmintos resultaron poco significativas debido al reducido número de casos diagnosticados. De hecho, se puede afirmar que hay pocas helmintosis en la comunidad evaluada.

Con relación a las técnicas diagnósticas empleadas, llama la atención el bajo rendimiento del método de formol-éter, para el diagnóstico global de los parásitos y especialmente para el diagnóstico de los protozoarios. Este método no superó el rendimiento del examen directo, aunque se sabe que es un método de concentración ampliamente utilizado y de eficacia comprobada (20). Posiblemente esto se deba al gran número de casos con formas vacuolares de $B$. hominis que fueron diagnosticadas. Estas fases evolutivas son muy frágiles y la manipulación que implica la técnica de formol-éter pudiera determinar su destrucción.

El examen directo es la técnica estándar usada en la mayoría de los laboratorios de Venezuela y se utiliza ampliamente en estudios epidemiológicos (25). Este método fue el más apropiado para la identificación de protozoarios, especialmente de $B$. hominis, que fue el parásito más común en la población evaluada. Se sabe que por razones de su biología el examen directo es el mejor método diagnóstico para este protozoario $(25,38)$.

Por otro lado, la técnica de Kato es un método que ha demostrado su eficacia para el diagnóstico de helmintos como A. lumbricoides, T. trichiura y ancilostomideos, entre otros (39). Hecho que fue comprobado en el presente estudio ya que los helmintos diagnosticados con esta técnica casi duplicaron a los diagnosticados con el examen directo.

La elevada prevalencia de parasitosis intestinal encontrada no sólo obedece a factores ecológicos, sino también a factores socio-sanitarios, ya que se pudo verificar que la comunidad de Aripao presenta deficiencias en el saneamiento ambiental 


\section{R Devera, V Angulo, E Amaro, M Finali, G Franceschi, Y Blanco, R Maria Tedesco y col.}

básico. Además, las condiciones económicas y el nivel educativo no son de los mejores (datos no presentados). Todo esto engloba lo que ha sido llamado por algunos autores la etiología social de las parasitosis intestinales $(5,36)$.

En conclusión, se determinó una elevada prevalencia de parásitos intestinales (83.9\%) en habitantes de la comunidad rural de Aripao, municipio de Sucre, Estado Bolívar, sin predilección por el sexo o la edad, con predominio de los protozoarios, en particular de $B$. hominis (60\%).

Los resultados de los exámenes realizados fueron entregados a cada persona, así como un informe a las autoridades sanitarias competentes. Todos los parasitados recibieron de forma gratuita los medicamentos antiparasitarios específicos en cada caso.

\section{AGRADECIMIENTOS.}

Este trabajo fue financiado por el Consejo de Investigación de la Universidad de Oriente, Proyectos CI-20407-1165/04 y CI-2-0407-1130/03. Nuestro agradecimiento al Alcalde y a todo el personal de la alcaldía del municipio de Sucre por el apoyo prestado; a la Sra. María y demás enfermeras del Ambulatorio Rural Tipo I de Aripao por su colaboración y hospitalidad. Al Sr. Aníbal Hernández por todo el apoyo logístico. Al Colegio de Médicos del Estado Bolívar por la donación de medicamentos empleados para tratar a los parasitados. A los habitantes de Aripao que aceptaron participar en el estudio y por habernos recibido tan cordialmente.

\section{REFERENCIAS.}

1.- OMS. Infecciones intestinales por protozoos y helmintos. Ginebra, Edit. Gráficas Reunidas; 1981. Serie informes técnicos 666. p 155.

2.- Savioli L, Bundy DAP, Tomkins A. Intestinal parasitic infections: a soluble public health problem. Trans R Soc Trop Med Hyg 1992; 86:353-4.

3.- Chan MS. The global burden of intestinal nematode infections-fifty years on. Parasitol Today 1997; 13: 43843.

\section{Revista Biomédica}

4.- Botero D. Persistencia de parasitosis intestinales endémicas en América Latina. Bull Of Sanit Panam 1981; 90:39-47.

5.- Chacín Bonilla L. El problema de las parasitosis intestinales en Venezuela. Invest Clin 1990; 31: 1-2.

6.- World Health Oranization. WHO Expert Committee. Public health significance of intestinal parasitic infections. Bull WHO 1987; 65:575-88.

7.- Torres P, Miranda JC, Duran L, Riquelme JM, Franjola $\mathrm{R}$, Perez J, et al. Blastocistosis y otras infecciones por protozoarios intestinales en comunidades humanas ribereñas de la cuenca del río Valdivia, Chile. Rev Inst Med Trop São Paulo 1992; 34:557-64.

8.- Kobayashi J, Hasegawa H, Forli A, Nishimura N, Yamanaka A, Shimabukuro T, et al. Prevalence of intestinal parasitic infection in five farms in Holambra, São Paulo, Brazil. Rev Inst Med Trop São Paulo 1995; 37:13-8.

9.- Ramos L, Salazar-Lugo R. Infestación parasitaria en niños de Cariaco-Estado Sucre, Venezuela y su relación con las condiciones socio-económicas. Kasmera 1997; 25:175-89.

10.- Urdaneta H, Cova JA, Alfonzo N, Hernández M. Prevalencia de enteroparásitos en una comunidad rural venezolana. Kasmera 1999; 27:41-51.

11.- Devera R, Cermeño, Blanco Y, Bello Montes MC, Guerra X, De Sousa M, et al. Prevalencia de blastocistosis y otras parasitosis intestinales en una comunidad rural del Estado Anzoátegui, Venezuela. Parasitol Latinoamer 2003; 58: 95-100.

12.- Devera R, Requena I, Velasquez V, Castillo H, Guevara $\mathrm{R}$, De Sousa M, et al. Balantidiasis en una comunidad rural del estado Bolívar, Venezuela. Bol Chil Parasitol 1999; 54: 7-12.

13.- Cedeño J. Parasitosis intestinales en estudiantes de la Escuela Rural "Dr. José María Vargas”, Cambalache, Puerto Ordaz, estado Bolívar. [Tesis] Dpto. Parasitología y Microbiología, Universidad de Oriente, 2001.

14.- Mago Y. Parasitosis Intestinales en niños de un Barrio de El Callao, Estado Bolívar. [Tesis] Departamento de Parasitología y Microbiología, Universidad de Oriente; 2004 


\section{Parásitos intestinales en una comunidad rural}

15.- Botero D, Restrepo M. Parasitosis humanas. $1^{\text {a }}$ ed. Medellín: Corporación para Investigaciones Biológicas; 1998. p. 457.

16.- Morales G, Pino LA. Parasitología cuantitativa. Caracas, Fondo Editorial Acta Científica Venezolana; 1987.

17.- Díaz I, Duran TF. Prevalencia de parasitosis intestinales en alumnos de educación básica del Municipio Cacique Mara, Maracaibo-Estado Zulia. Kasmera 1990; 18:46-71.

18.- Chacín Bonilla L, Bonilla E, Parra AM, Estevez J, Morales LM, Suarez H. Prevalence of Entamoeba histolytica and other intestinal parasites in a community from Maracaibo, Venezuela. Ann Trop Med Parasitol 1992; 86: 373-80.

19.- Rivero Rodríguez Z, Chango Gómez Y, Iriarte Nava H. Enteroparásitos en alumnos de la Escuela Básica Dr. “Jesús María Portillo”, Municipio Maracaibo, Edo. Zulia, Venezuela. Kasmera 1997; 25:121-8.

20.- Rivero Rodríguez Z, Chourio-Lozano G, Díaz I, Cheng R, Rucson G. Enteroparásitos en escolares de una institución pública del municipio Maracaibo, Venezuela. Invest Clin 2000; 41:37-57.

21.- Morales GA, Pino L, Artega C, Matinella L, Rojas $\mathrm{H}$. Prevalencias de las geohelmintiasis intestinales en 100 municipios de Venezuela (1898-1992). Rev Soc Bras Med Trop 1999; 32:263-70.

22.- Al Rumhein F, Sánchez J, Requena I, Blanco Y, Devera R. Parasitosis intestinales en escolares: relación entre su prevalencia en heces y en el lecho subungueal. Rev Biomed 2005; 16: 227-37.

23.- Tutaya R, Blanco Y, Sandoval M, Alcala F, Aponte M, Devera R. Coccidios intestinales en habitantes del Barrio 6 de Noviembre, Ciudad Bolívar, Estado Bolívar, Venezuela. Rev Biomed 2006; 17: 152-4.

24.- Devera R, Nastasi J, Niebla G, González R, Velásquez, V. Prevalencia de infección por Blastocystis hominis en escolares de Ciudad Bolívar, Venezuela. Bol Chil Parasitol 1997; 52:77-81.

25.-Devera R, Niebla G, Nastasi J, Velásquez V, González $\mathrm{R}$. Valor del examen directo de heces en el diagnóstico de Blastocystis hominis. Memórias del XVI Congresso Brasileiro de Parasitologia. Poços de Caldas; 1999. p. 201.
26.- Arcay L, Borges E, Bruzual E. Incidencia de Cyclospora cayetanensis y Microspora en pacientes de la población circunvecina del Río Anare (Venezuela) y su presencia en el agua del río. Arch Hosp Vargas 1998; 40: 157-62.

27.- Baraldi S, Marques E, Dias R. Ocorrencia de Cryptosporidium parvum e Isospora belli na regiáo de Campinas, SP. Rev Inst Adolfo Lutz 1999; 58:97-103.

28.- Arcay L, Bruzual E. Cryptosporidium en ríos de Venezuela. Encuesta epidemiológica de una población humana y fauna en convivencia. Parasitol dia 1993; 17: 11-8.

29.- Chacín Bonilla L, Mejía de Young M, Cano G, Guanipa N, Estévez J, Bonilla E. Cryptosporidium infections in a suburban community in Maracaibo, Venezuela. Am J Trop Med Hyg 1993; 49:63-7.

30.- Chacín Bonilla L, Mejía de Young M, Estévez J. Prevalence and pathogenic role of Cyclospora cayetanensis in a Venezuelan community. Am J Trop Med Hyg 2003; 68:304-6.

31.- Devera R, Blanco Y, Cabello E. Elevada prevalencia de Cyclospora cayetanensis en indígenas del estado Bolívar, Venezuela. Cad Saúde Púb 2005; 21:1778-84.

32.- Chacín Bonilla L, Guanipa N, Cano G, Raleigh X, Quijada L. Criptosporidiosis among patients with adquired immunodeficiency syndrome in Zulia State, Venezuela. Am J Trop Med Hyg 1992; 47:582-6.

33.- Costa-Cruz J, Ferreira M, Rossin I. Intestinal parasites in AIDS and +HIV patients in Uberlândia, Minas Gerais, Brazil. Mem Inst Oswaldo Cruz 1996; 91:685-6.

34.- Chacín Bonilla L, Bonilla MC, Soto-Torres L, RiosCandida Y, Sardina M, Enmanuels C, et al. Cryptosporidium parvum in children with diarrhea in Zulia State, Venezuela. Am J Trop Med Hyg 1997; 56:365-9.

35.- Cermeño J, Hernández I, Uzcátegui O, Páez J, Rivera $\mathrm{M}$, Baliachi N. Parasitosis intestinal en pacientes infectados por el virus de inmunodeficiencia humana. Kasmera 2004; 32:101-7.

36.- Devera R, Niebla PG, Nastassi CJ, Velásquez AV, González MR. Prevalencia de Trichuris trichiura y otros enteroparásitos en siete escuelas del área urbana de Ciudad Bolívar, Estado Bolívar, Venezuela. Saber 2000; 12:41-7. 
R Devera, V Angulo, E Amaro, M Finali, G Franceschi, Y Blanco, R Maria Tedesco y col.

37.- Penott A, Chinchilla O. Prevalencia de estrongiloidiosis y evaluación de la eficacia del albendazol e ivermectina en individuos provenientes de la comunidad de Santa Fé, estado Sucre, Venezuela. Saber; 1995; 8:46-9.

38.- Castilho VP, Goncalves EN, Vieira GC, Vergilio DR, Uemura IH, Faria VA, et al. Blastocystis hominis: investigação laboratorial. J Bras Patol 1998; 34:240.

39.- Katz N, Chavez A, Pellegrino, J. A simple device for quantitative stool thick-smear technique in Schistosomiasis mansoni. Rev Inst Med Trop São Paulo 1972; 14:397400. 